\title{
BMJ Open Non-inferiority trials: are they inferior? A systematic review of reporting in major medical journals
}

\author{
Sunita Rehal, ${ }^{1,2}$ Tim P Morris, ${ }^{1,2}$ Katherine Fielding, ${ }^{1,3}$ James R Carpenter, ${ }^{1,2,4}$ \\ Patrick P J Phillips ${ }^{1}$
}

To cite: Rehal S, Morris TP, Fielding $\mathrm{K}$, et al. Noninferiority trials: are they inferior? A systematic review of reporting in major medical journals. BMJ Open 2016;6: e012594. doi:10.1136/ bmjopen-2016-012594

- Prepublication history and additional material is available. To view please visit the journal (http://dx.doi.org/ 10.1136/bmjopen-2016012594).

Received 11 May 2016 Revised 29 July 2016 Accepted 16 September 2016

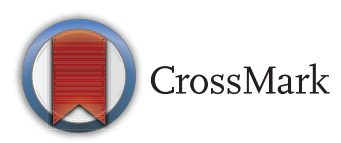

For numbered affiliations see end of article.

Correspondence to

Sunita Rehal;

s.rehal@ucl.ac.uk

\section{ABSTRACT}

Objective: To assess the adequacy of reporting of noninferiority trials alongside the consistency and utility of current recommended analyses and guidelines.

Design: Review of randomised clinical trials that used a non-inferiority design published between January 2010 and May 2015 in medical journals that had an impact factor > 10 (JAMA Internal Medicine, Archives Internal Medicine, PLOS Medicine, Annals of Internal Medicine, BMJ, JAMA, Lancet and New England Journal of Medicine).

Data sources: Ovid (MEDLINE).

Methods: We searched for non-inferiority trials and assessed the following: choice of non-inferiority margin and justification of margin; power and significance level for sample size; patient population used and how this was defined; any missing data methods used and assumptions declared and any sensitivity analyses used. Results: A total of 168 trial publications were included. Most trials concluded non-inferiority (132; 79\%). The non-inferiority margin was reported for $98 \%$ (164), but less than half reported any justification for the margin $(77 ; 46 \%)$. While most chose two different analyses $(91 ; 54 \%)$ the most common being intention-to-treat (ITT) or modified ITT and per-protocol, a large number of articles only chose to conduct and report one analysis $(65 ; 39 \%)$, most commonly the ITT analysis. There was lack of clarity or inconsistency between the type I error rate and corresponding Cls for 73 (43\%) articles. Missing data were rarely considered with (99; $59 \%$ ) not declaring whether imputation techniques were used.

Conclusions: Reporting and conduct of non-inferiority trials is inconsistent and does not follow the recommendations in available statistical guidelines, which are not wholly consistent themselves. Authors should clearly describe the methods used and provide clear descriptions of and justifications for their design and primary analysis. Failure to do this risks misleading conclusions being drawn, with consequent effects on clinical practice.

\section{INTRODUCTION}

Non-inferiority trials assess whether a new intervention is not much worse when

\section{Strengths and limitations of this study}

- This research clearly demonstrates the inconsistency in recommendations for non-inferiority trials provided by guidelines for researchers and this is reflected within this review.

- It highlights missing data and sensitivity analyses in the context of non-inferiority trials.

- It provides recommendations using examples for researchers using the non-inferiority design.

- Justification of the choice of the margin was recorded as such if any attempt was made to do so, and so one could argue that inadequate attempts were counted as a 'justification'; however, there was good agreement between reviewers when independently assessed.

- Only one reviewer extracted information from all articles and therefore assessments may be subjective. However, there was good agreement when a random $5 \%$ of papers were independently assessed.

compared to a standard treatment or care. These trials answer whether we are willing to accept a new intervention that may be clinically worse, yet still be beneficial for patients while having another advantage, such as less-intensive treatment, lower cost or fewer side effects. ${ }^{1}$ Non-inferiority and equivalence are sometimes, mistakenly, used interchangeably. Equivalence trials are designed to show that a new intervention performs not much worse and not much better than a standard intervention. Both trial designs are different to superiority trials, which aim to show that a new intervention performs better when compared to a control.

Poor trial quality can bias trial results towards concluding no difference between treatments. ${ }^{2}$ This creates more challenges in non-inferiority trials than superiority trials as such bias can produce false-positive results for non-inferiority. ${ }^{3-5}$ The increasing use of this design ${ }^{6-8}$ means that it is even more important for trialists to understand the 
issues around the quality in the design and analysis of non-inferiority trials.

There are several guidelines available to aid researchers using a non-inferiority design, where various considerations of the design are explained and discussed (table 1).

1. The CONSORT extension statements ${ }^{1}{ }^{9}$ focus on the reporting of non-inferiority trials, with the most recent 2012 statement being an elaboration of the 2006 statement.

2. The draft FDA $2010^{2}$ document focuses on all aspects and issues relative to non-inferiority trials and gives general guidance.

3. The EMEA 2000 guideline $^{10}$ discusses switching between non-inferiority and superiority designs and the EMEA $2006^{11}$ guideline discusses the choice of the non-inferiority margin, taking into account two-arm and three-arm trials.

4. The ICH E9 and E10 guidelines ${ }^{12} 13$ are general statistical guidance documents addressing issues for all clinical trials and designs.

5. SPIRIT $^{14}$ is a guidance document for protocols for all trial designs and includes discussions of recently developed methodology.

There is some inconsistency between these guidelines regarding the conduct of non-inferiority trials (table 1) that may adversely affect the overall quality and reporting of non-inferiority trials. Non-inferiority trials require more care around certain issues, and so clear guidance on how to design and analyse these trials are necessary. Some of these issues that can influence inferences made about non-inferiority are outlined below.

First, the non-inferiority margin-the value that allows for a new treatment to be "acceptably worse"1 —is used as a reference for conclusions about non-inferiority. It is recommended by all guidelines that this margin is chosen on a clinical basis, meaning the maximum clinically acceptable extent to which a new drug can be less effective than the standard of care and still show evidence of an effect. ${ }^{15}$ However, it is unclear whether statistical considerations should also affect the choice of an appropriate margin, as recommended by the Draft FDA 2010, ICH E10 and EMEA 2006 guidelines ${ }^{2} 11 \quad 13$ (table 1). Ignoring statistical evidence from meta-analyses or systematic reviews could have the potential for researchers to choose an unrealistic margin.

Second, it is important to choose who is included in analyses for non-inferiority trials. The intention-to-treat (ITT) analysis (includes all randomised patients irrespective of postrandomisation occurrences) is preferred for superiority trials as it is likely to lead to a treatment effect closer to having no effect and so is conservative. ${ }^{16}$ For non-inferiority trials, the ITT analysis can bias towards the null, which may lead to false claims of noninferiority. ${ }^{17}$ The alternative per-protocol (PP) analysis is often considered instead. However, given that the PP analysis allows for the exclusion of patients, it fails to preserve a balance of patient numbers between treatment arms (ie, randomisation) that ITT analysis does and can cause bias in either direction, depending on who the analysis excludes. ${ }^{18}$ Guidelines often recommend performing the ITT and PP analyses, although definitions are inconsistent (table 1). In particular, the CONSORT 2006 guidelines describe the PP analysis as excluding patients not taking allocated treatment or otherwise not protocol-adherent, ${ }^{1}$ whereas the ICH E9 guidelines state that the $\mathrm{PP}$ analysis is a "subset of patients who complied sufficiently with the protocol, such as exposure to treatment, availability of measures and absence of major protocol violations." 19 These obscure definitions could lead researchers to arbitrarily exclude patients from analyses. The draft FDA guidelines recommend researchers to use an ITT and as-treated analysis, although it is unclear what is meant by 'as-treated' as this is not defined within the guidelines. Other frequently used classifications such as modified ITT (mITT), which aims to contain 'justifiable' exclusions (eg, patients who never had the disease of interest) from the ITT analysis, are also defined inconsistently. ${ }^{20}$ Third, while two-sided $95 \%$ CIs are widely used for superiority trials, there is some inconsistent advice as whether to calculate $90 \%$ or $95 \%$ CIs for noninferiority trials and whether these should be presented as one-sided or two-sided intervals (table 1).

Fourth, the handling of missing data is generally discussed for all trials but rarely in the specific context of non-inferiority trials. Methods recommended to handle missing data vary between guidelines. The ICH E9 guidelines recommend using a last observation carried forward imputation method, ${ }^{19}$ and the more recent SPIRIT guidelines recommend multiple imputation, but caution the reader that it relies on untestable assumptions $^{14}$ (table 1). Methods to handle missing data often contain untestable assumptions and so, sensitivity analyses are essential to test the robustness of conclusions under different assumptions. ${ }^{12}$ However, it is unclear what sensitivity analyses are appropriate for noninferiority trials.

Given the inconsistency between guidelines, we hypothesised that poor conduct and reporting would be associated with demonstrating non-inferiority. This review investigates the quality of conduct and reporting for non-inferiority trials in a selection of high-impact journals over a 5-year period. We also provide recommendations to aid trialists who may consider a noninferiority design.

\section{METHODS}

Medical journals (general and internal medicine) with an impact factor $>10$ according to the ISI web of knowledge $^{21}$ were included in the review (correct at time of search on 31 May 2015), the rationale being that articles published in these journals are likely to have the highest influence on clinical practice and be the most rigorously 
Table 1 Summary of guidelines

\begin{tabular}{|c|c|c|c|c|c|}
\hline & Justification of margin & Who is included in analysis & $\mathrm{Cl}$ & Missing data & Sensitivity analyses \\
\hline CONSORT $2006^{1}$ & $\begin{array}{l}\text { 'Margin should be } \\
\text { specified and preferably } \\
\text { justified on clinical } \\
\text { grounds' }\end{array}$ & $\begin{array}{l}\text { 'Non-ITT analyses might be } \\
\text { desirable as a protection from } \\
\text { ITTs increase in type I error. } \\
\text { There is greater confidence in } \\
\text { results when the conclusions are } \\
\text { consistent'. } \\
\text { ITT: 'Analysing all patients within } \\
\text { their randomised groups, } \\
\text { regardless of whether they } \\
\text { completed allocated treatment is } \\
\text { recommended' } \\
P P \text { : 'Alternative analyses that } \\
\text { exclude patients not taking } \\
\text { allocated treatment or otherwise } \\
\text { not protocol-adherent could bias } \\
\text { the trial in either direction. The } \\
\text { terms on-treatment or PP } \\
\text { analysis are often used but may } \\
\text { be inadequately defined'. }\end{array}$ & $\begin{array}{l}\text { 'Many non-inferiority } \\
\text { trials based their } \\
\text { interpretation on the upper } \\
\text { limit of a one-sided } 97.5 \% \\
\mathrm{CI} \text {, which is the same as } \\
\text { the upper limit of a } \\
\text { two-sided } 95 \% \mathrm{Cl} \text { '. } \\
\text { 'Although one-sided and } \\
\text { two-sided Cls allow for } \\
\text { inferences about } \\
\text { non-inferiority, we suggest } \\
\text { that two-sided Cls are } \\
\text { appropriate in most } \\
\text { non-inferiority trials. If a } \\
\text { one-sided } 5 \% \text { significance } \\
\text { level is deemed } \\
\text { acceptable for the } \\
\text { non-inferiority hypothesis } \\
\text { test (a decision open to } \\
\text { question), a } 90 \% \\
\text { two-sided Cl could then be } \\
\text { used'. }\end{array}$ & & \\
\hline CONSORT $2012^{9}$ & & $\begin{array}{l}\text { 'Should be indicated if } \\
\text { conclusions are related to PP } \\
\text { analysis, ITT analysis or both } \\
\text { and if the conclusions are stable } \\
\text { between them'. }\end{array}$ & $\begin{array}{l}\text { 'The two-sided } \mathrm{Cl} \text { provides } \\
\text { additional information, in } \\
\text { particular for the situation } \\
\text { in which the new treatment } \\
\text { is superior to the reference } \\
\text { treatment' }\end{array}$ & & $\begin{array}{l}\text { Sensitivity analysis is } \\
\text { discussed through an } \\
\text { example: 'Study endpoints } \\
\text { were analysed primarily for } \\
\text { the PP population and } \\
\text { repeated, for sensitivity } \\
\text { reasons, for the ITT } \\
\text { population'. }\end{array}$ \\
\hline Draft FDA $2010^{2}$ & $\begin{array}{l}\text { 'Whether M1 (the effect of } \\
\text { the active control arm } \\
\text { relative to placebo) is } \\
\text { based on a single study or } \\
\text { multiple studies, the } \\
\text { observed (if there were } \\
\text { multiple studies) or } \\
\text { anticipated (if there is only } \\
\text { one study) statistical } \\
\text { variation of the treatment } \\
\text { effect size should }\end{array}$ & $\begin{array}{l}\text { 'It is therefore important to } \\
\text { conduct both ITT and } \\
\text { "as-treated" analyses in } \\
\text { non-inferiority studies'. } \\
\text { ITT: 'preserve the principle that } \\
\text { all patients are analysed } \\
\text { according to the treatment to } \\
\text { which they have been } \\
\text { randomised even if they do not } \\
\text { receive it' }\end{array}$ & $\begin{array}{l}\text { 'Typically, the one-sided } \\
\text { type I error is set at } 0.025 \text {, } \\
\text { by asking that the upper } \\
\text { bound of the } 95 \% \mathrm{Cl} \text { for } \\
\text { control treat be less than } \\
\text { the } \mathrm{NI} \text { margin. If multiple } \\
\text { studies provide very } \\
\text { homogeneous results for } \\
\text { one or more important } \\
\text { endpoints, it may be } \\
\text { possible to use the } 90 \%\end{array}$ & $\begin{array}{l}\text { 'Poor quality can reduce } \\
\text { the drug's effect size } \\
\text { and undermine the } \\
\text { assumption of the effect } \\
\text { size of the control agent, } \\
\text { giving the study a "bias } \\
\text { towards the null"'. }\end{array}$ & \\
\hline
\end{tabular}


Justification of margin

contribute to the ultimate choice of M1, as should any concerns about

constancy. The selection

of M2 (the largest

clinically acceptable

difference of the test

treatment compared to the

active control) is then

based on clinical

judgment regarding how

much of the M1 active

comparator treatment

effect can be lost. The

exercise of clinical

judgment for the

determination of $\mathrm{M} 2$

should be applied after

the determination of M1

has been made based on

the historical data and

subsequent analysis'

$\mathrm{ICH} \mathrm{E9}{ }^{12}$ 'This margin is the largest difference that can be judged as being clinically acceptable'

\section{Who is included in analysis}

Who is included in analysis

lower bound rather than the $95 \%$ lower bound of the $\mathrm{Cl}$ to determine the active control effect size

'In confirmatory trials, it is usually 'For non-inferiority trials, a appropriate to plan to conduct an one-sided interval should analysis of the full analysis set and a PP analysis. In an equivalence or non-inferiority trial, use of the full analysis set is generally not conservative and its role should be considered very carefully'.

ITT: 'participants allocated to a treatment group should be followed up, assessed and analysed as members of that group irrespective of their compliance to the planned course of treatment'.

Full analysis set: 'The set of participants that is as close as possible to the ideal implied by from the use of a
Imputation techniques, ranging from LOCF to the use of complex mathematical models, may be used to compensate for missing data one-sided or two-sided procedure'.
'An investigation should be made concerning the sensitivity of the results of analysis to the method of handling missing values, especially if the number of missing values is substantial'. 
the ITT principle. It is derived from the set of all randomised participants by minimal and justified elimination of participants'.

$P P$ : 'The set of data generated

by the subset of participants who complied with the protocol sufficiently to ensure that these data would be likely to exhibit the effects of treatment, according to the underlying scientific model.

Compliance covers such considerations as exposure to treatment, availability of measurements and absence of major protocol violations'.
$\mathrm{ICH} \mathrm{E} 10^{13}$
'The determination of the margin in a non-inferiority trial is based on statistical reasoning and clinical judgment'

Use an example where

'non-inferiority would be claimed if ITT and PP analyses show conclusions of NI'.

ITT: 'To preserve the unique benefit of randomisation as a mechanism to avoid selection bias, an "as randomised' analysis retains participants in the group to which they were originally allocated. To prevent attrition bias, outcome data obtained from all participants are included in the data analysis,

regardless of protocol

adherence'.

$P P$ and mITT: 'Some trialists use other types of data analyses
'Multiple imputation can be used to handle missing data although relies on untestable assumptions'
'Sensitivity analyses are highly recommended to assess the robustness of trial results under different methods of handling missing data' 
(commonly labelled as "mITT" or

"PP") that exclude data from certain participants-such as those who are found to be

ineligible after randomisation or who deviate from the intervention or follow-up protocols. This exclusion of data from protocol non-adherers can introduce bias, particularly if the frequency of and the reasons for

non-adherence vary between the study groups'.

EMEA $2006^{11} \quad$ 'The choice of delta must always be justified on clinical and statistical grounds'
EMEA $2000^{10}$

'A two-sided $95 \% \mathrm{Cl}$ (or one-sided $97.5 \% \mathrm{Cl}$ ) is constructed. The interval should lie entirely on the positive side of the margin. Statistical significance is generally assessed using the two-sided 0.05 level of significance (or one-sided 0.025)'

'ITT and PP analyses have equal 'A two-sided $\mathrm{Cl}$ should lie importance and their use should entirely to the right of

lead to similar conclusions for

delta. If one-sided

confidence is used then $97.5 \%$ should be used'

'It will be necessary to pay particular attention to

demonstrating the

sensitivity of the trial by showing similar results for the full analysis set and PP analysis set' 
conducted and reported due to the thorough editorial process. We searched Ovid (MEDLINE) using the search terms 'noninferior', 'non-inferior', 'noninferiority' and 'non-inferiority' in titles and abstracts between 1 January 2010 and 31 May 2015 in New England Journal of Medicine (NEJM), Lancet, JAMA, British Medical Journal, Annals of Internal Medicine, PLOS Medicine and Archives of Internal Medicine (descending impact order). From 2013, Archives of Internal Medicine was renamed JAMA Internal Medicine, and, therefore, both journals have been included in this review. All journals refer authors to the CONSORT statement and checklist when reporting. Eligibility of articles was assessed via abstracts by two reviewers (SR and TPM). Articles included were non-inferiority randomised controlled clinical trials. Articles were excluded if the primary analysis was not for non-inferiority. Systematic reviews, meta-analyses and commentaries were also excluded. Few trials were designed and analysed using Bayesian methods and were therefore excluded for consistent comparability in frequentist methods.

Before performing the review, a data extraction form was developed to extract information from articles. Information extracted was with regard to the primary outcome. The form was standardised to collect information on the year of publication, non-inferiority margin (and how the margin was justified), randomisation, type of intervention, disease area, sample size, analyses performed (how this was defined and what was classed as primary/secondary), primary outcome, $\mathrm{p}$ values (and whether this was for a superiority hypothesis), significance level of CIs (and whether both bounds were reported), imputation techniques for missing data, sensitivity analyses, conclusions of non-inferiority and whether a test for superiority was prespecified. Justifications for the choice of the non-inferiority margin were reviewed by two reviewers (SR and PPJP). See online supplementary material for further details on methods.

A quality grading system was developed based on whether the margin was justified (yes vs no/poor), how many analyses were performed on the primary outcome $(<2$ vs $\geq 2)$ and whether the type I error rate was consistent with the significance level of the CI (yes vs no/ unclear). Articles were classed as 'excellent' if all these criteria were fulfilled and were classed as 'poor' if none was fulfilled. Articles which satisfied one criterion were classed as 'fair' and articles that provided two of the three criteria were classified as 'good'. The results of this grading were compared to inferences on noninferiority to assess if the quality of reporting was associated with concluding non-inferiority at the 5\% significance level.

Additional published online supplementary material was accessed only if it specifically referred to the information we were extracting within articles. As a substudy, all statistical methods, outcomes and sample sizes from protocols and/or online supplementary material were reviewed from $N E J M$ as the journal is known to specifically request and publish protocols and statistical analysis plans alongside accepted publications.

Assessments were carried out by one reviewer (SR), with a random selection of $5 \%$ independently reviewed (PPJP). Any assessments that required a second opinion were independently reviewed (TPM). Any discrepancies were resolved by discussion between reviewers.

All analyses were conducted using Stata V.14.

\section{RESULTS}

Our search found 252 articles. After duplicate publications were removed, 217 were screened for eligibility using their titles and abstracts. A total of 46 articles were excluded leaving 171 articles to be reviewed. A further three articles were excluded during the full-text review leaving 168 articles (figure 1).

General characteristics of the included studies are summarised in table 2.

\section{Margin}

The non-inferiority margin was specified in 164 (98\%) articles and was justified in less than half of articles 76 (45\%). The most common justification was on a clinical basis $(29(17 \%))$, which was often worded ambiguously and with little detail. A total of $14(8 \%)$ used previous findings from past trials or statistical reviews to justify the choice of the margin (table 3 ).

\section{Patients included in analysis}

Over a third of articles $65(39 \%)$ declared only one analysis (table 3 and see online supplementary table S1a). The majority of trials classed ITT analysis as primary and PP analyses as secondary (see online supplementary figure S1a). PP analyses were performed in 90 (54\%) trials; of which, $11(12 \%)$ did not define what was meant by 'PP' (table 3 and see online supplementary table S1b). Definitions of the PP population contained various exclusions, mostly regarding errors in randomised treatment or treatment received.

\section{Type I error rate}

Consistency between the type I error rate and CIs reported was moderate at $95(57 \%)$ (table 4$)$. Most articles, $69(41 \%)$, used a one-sided $2.5 \%$ or (numerically equivalent) two-sided 5\% significance level (table 5) and some used a one-sided 5\% significance level, $46(27 \%)$. The majority of articles presented two-sided CIs (147; $88 \%)$ and $19(11 \%)$ articles presented one-sided CIs. Most two-sided CIs were at the 95\% significance level: $125(74 \%)$.

\section{Missing data and sensitivity analyses}

Ninety-nine $(59 \%)$ trials did not report whether or not any imputation was carried out and only 12 (7\%) explicitly declared that no imputation was used. Assuming a worst-case scenario or multiple imputation were the 
Figure 1 Flow chart of eligibility of articles.

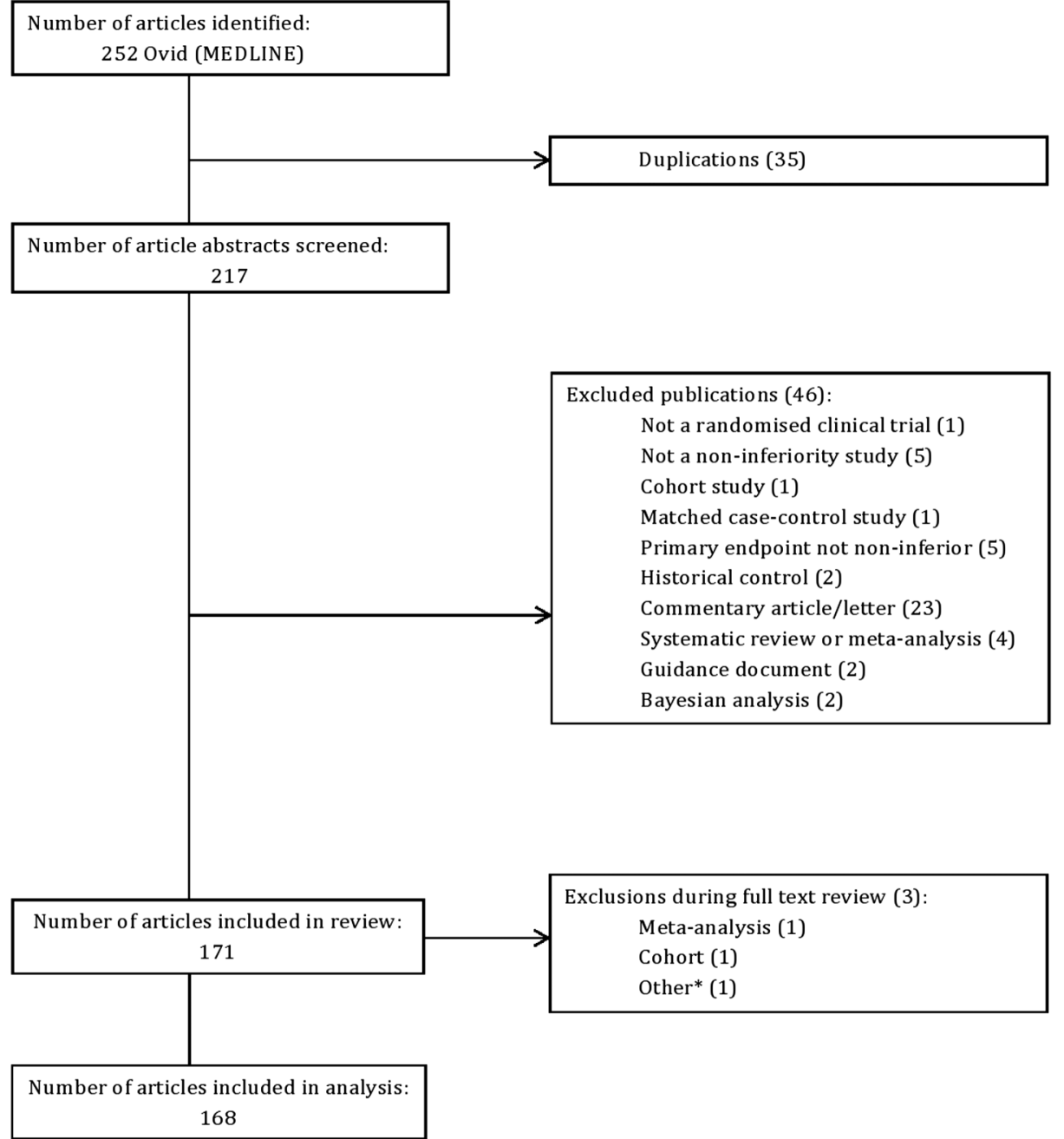

* Secondary analyses. Primary analyses for the same study was included in the review

most common methods used (table 6). The number of imputations used for multiple imputation was specified in 8 of 11 articles and 4 of 11 stated at least one of the assumptions from Rubin's rules. ${ }^{22}$ Sixty-four $(38 \%)$ trials reported using sensitivity analyses to test robustness of conclusions of the primary outcome; of these, 27 (42\%) were related to assumptions about the missing data (table 6).

\section{Study conclusions}

There were seven (4\%) articles that could not make definitive conclusions (noted as 'other'; table 7). For example, if all analyses conducted had to demonstrate non-inferiority to conclude a treatment was non-inferior, and only one of the analyses did, then non-inferiority could not be concluded and could not be rejected. Non-inferiority was declared in $132(79 \%)$ articles. Ten of these had made some reference with equivalence studies within the article (see online supplementary material).

Superiority analyses were performed in 37 (22\%) trials after declaring non-inferiority; of which, $27(73 \%)$ had explicitly preplanned for superiority analyses. p Values were reported in 98 (58\%) articles; of which, $29(30 \%)$ were testing a superiority hypothesis.

\section{Subgroup of trials with published protocols}

Additional information from protocols published by NEJM was extracted for 57 of 61 articles. Including this additional information provided by $N E J M$ improved reporting of results across all criteria: 39 (64\%) articles justified the choice of the non-inferiority margin compared to $19(31 \%)$; most planned two or more analyses $45(74 \%)$ compared to $37(61 \%)$ (there were a couple of cases where two analyses were planned in the protocol but only one was stated in the published article); consistency between type I error rates and CIs was 44 (72\%) compared with 36 (59\%); imputation techniques were considered in 29 (48\%) compared with $17(28 \%)$ articles and sensitivity analyses were considered in $38(62 \%)$ articles compared with $25(41 \%)$. The majority of articles concluded non-inferiority with $8(13 \%)$ not determining non-inferiority. A total of $14(23 \%)$ articles concluded superiority, of which most were pre- 


\section{Table 2 General characteristics}

\section{Characteristics}

Journal

NEJM

Lancet

JAMA

BMJ

Annals of Internal Medicine

PLoS Medicine

Archives of Internal Medicine

JAMA of Internal Medicine

Year of publication

2010

2011

2012

2013

2014

2015

Type of intervention

Drug

Surgery

Other

Randomisation

Patient

Cluster

Power

$80 \%$

$85 \%$

$90 \%$

71-99\% (excluding the above)

Not reported/unclear

Composite outcome

Yes

No

Disease

Heart disease

Blood disorder

Cancer

Diabetes

Thromboembolism

Skin infection (non-contagious)

Urinary tract infection

Arthritis

Opthomology

Pneumonia

Complications in pregnancy

Stroke

Testing method

Appendicitis

Depression

Other non-infectious disease

HIV

Tuberculosis

Malaria

Skin infection (contagious)

Hepatitis C

Other infectious disease
All articles $(n=168)$

n (\%)

Including NEJM

protocols $(\mathrm{n}=61)$

n (\%)

$61(36)$

61

$19(11)$

8 (5)

$5(2)$

7 (4)

$2(1)$

2 (1)

$26(15)$

27 (16)

29 (17)

39 (23)

27 (16)

$20(12)$

$112(67)$

$22(13)$

$34(20)$

$163(97)$

5 (3)

9 (15)

9 (15)

8 (13)

$19(31)$

$10(16)$

$6(10)$

$44(72)$

7 (11)

10 (16)

$59(97)$

2 (3)

$6(36)$

11 (7)

65 (39)

$21(12)$

10 (6)

19 (31)

5 (8)

26 (43)

11 (18)

0

78 (46)

37 (61)

90 (54)

24 (39)

30 (18)

19 (11)

16 (10)

$13(21)$

6 (10)

8 (13)

11 (7)

6 (4)

3 (2)

3 (2)

3 (2)

3 (2)

3 (2)

3 (2)

3 (2)

2 (3)

6 (10)

2 (3)

0

1 (2)

1 (2)

1 (2)

0

2 (3)

3 (2)

2 (1)

2 (1)

18 (11)

18 (11)

6 (4)

4 (2)

2 (1)

1 (2)

1 (2)

0

7 (11)

2 (3)

4 (7)

1 (2)

0

2 (3)

2 (1)

8 (5) 
Table 3 Justification of choice of margin, total number of patient populations considered for analyses and patient population included in the analysis

\begin{tabular}{lcc}
\hline & $\begin{array}{c}\text { All articles } \\
\mathbf{( N = 1 6 8 )} \\
\mathbf{n}(\%)\end{array}$ & $\begin{array}{c}\text { Including NEJM } \\
\text { protocols (N=61) } \\
\mathbf{n} \text { (\%) }\end{array}$ \\
\hline $\begin{array}{l}\text { Justification of NI margin } \\
\text { Made no attempt for justification }\end{array}$ & $90(54)$ & $22(36)$ \\
Clinical basis. No evidence for consultation with external expert group, and no & $32(19)$ & $11(18)$ \\
reference to previous trials of the control arm & $13(8)$ & $14(23)$ \\
Preservation of treatment effect based on estimates of control arm effect from & & \\
previous trials & $6(4)$ & $3(5)$ \\
Expert group external to the authors. No reference to previous trials of the control & & \\
arm & $5(3$ & $2(3)$ \\
The same margin as was used in other similar trials & $4(2)$ & $1(2)$ \\
10-12\% recommended by disease-specific FDA guidelines & $4(2)$ & 0 \\
General comment that margin was decided according to FDA/regulatory guidance & 0 \\
Clinical basis and based on previous similar trial. No evidence for consultation with & $3(2)$ & \\
external expert group, and no reference to previous trials of the control arm & 0 & $2(3)$ \\
Based on registry/development programme & $11(7)$ & $6(10)$ \\
Other* & & $15(25)$ \\
Number of analyses & $65(39)$ & $38(62)$ \\
One & $91(54)$ & $7(11)$ \\
Two & $10(6)$ & $1(2)$ \\
Three & $2(1)$ & \\
Not defined & & $44(72)$ \\
Analysis & $129(77)$ & $35(57)$ \\
ITT & $90(54)$ & $17(28)$ \\
PP & $34(20)$ & $6(10)$ \\
mITT & $4(2)$ & $10(16)$ \\
As-treated & $20(12)$ & $2(3)$ \\
Other & $2(1)$ & \\
Unclear & & \\
*See online supplementary material. & & \\
ITT, intention to treat; mITT, modified intention to treat; PP, per-protocol. & & \\
& & \\
\end{tabular}

Table 4 Consistency of type I error rate with significance levels of Cls over year of publication

\begin{tabular}{|c|c|c|c|c|c|c|c|}
\hline & \multicolumn{7}{|c|}{ Year of publication } \\
\hline & 2010 & 2011 & 2012 & 2013 & 2014 & 2015 & Total \\
\hline \multicolumn{8}{|c|}{ All articles $(\mathrm{N}=168)$} \\
\hline Yes & $11(42 \%)$ & $15(56 \%)$ & $15(52 \%)$ & $24(62 \%)$ & $19(70 \%)$ & $11(55 \%)$ & $95(57 \%)$ \\
\hline No & 5 (19\%) & $4(15 \%)$ & $4(14 \%)$ & $5(13 \%)$ & $5(19 \%)$ & $3(15 \%)$ & $26(15 \%)$ \\
\hline Not reported & $10(38 \%)$ & $8(30 \%)$ & $10(34 \%)$ & $10(26 \%)$ & $3(11 \%)$ & $6(30 \%)$ & $47(28 \%)$ \\
\hline \multicolumn{8}{|c|}{ NEJM subgroup ( $\mathrm{N}=61)$} \\
\hline Yes & $7(78 \%)$ & $6(67 \%)$ & $5(63 \%)$ & $14(74 \%)$ & $8(80 \%)$ & $4(67 \%)$ & $44(72 \%)$ \\
\hline No & $1(11 \%)$ & 2 (22\%) & $2(25 \%)$ & $3(16 \%)$ & $2(20 \%)$ & $1(17 \%)$ & $11(18 \%)$ \\
\hline Not reported & $1(11 \%)$ & $1(11 \%)$ & $1(13 \%)$ & $2(11 \%)$ & 0 & $1(17 \%)$ & $6(10 \%)$ \\
\hline
\end{tabular}

planned $(9 ; 64 \%)$. Few articles $8 / 40(20 \%)$ presented superiority $\mathrm{p}$ values.

\section{Association between quality of reporting and conclusions}

Trials that were classed as having some 'other' conclusion about non-inferiority were excluded from the analysis. Overall, there was a suggestive difference between the quality of reporting and concluding non-inferiority: $\chi_{1}^{2}=3.76 ; \mathrm{p}=0.05$ (Cochran-Armitage test; table 7). Trials that were poorly reported were less likely to conclude non-inferiority than those that satisfied two or all criteria from justifying the choice of the margin, reporting two or more analyses or reporting a CI consistent with the type I error rate.

\section{DISCUSSION}

Reporting of non-inferiority trials is poor and is perhaps partly due to disagreement between guidelines on vital issues. There are some aspects that guidelines agree on, such as a requirement for the non-inferiority margin to 
Table 5 Significance level of (a) type I error rate and (b) $\mathrm{Cls}$ for all articles by whether $\mathrm{Cl}$ was one-sided or two-sided

\section{One-sided Two-sided Not reported}

(a) Type I error rate (\%)

$\begin{array}{llll}0.8 & 0 & 1(1 \%) & 0 \\ 1.25 & 3(2) & 0 & 0 \\ 2.45 & 1(1) & 0 & 0 \\ 2.5 & 40(24) & 2(1) & 2(1) \\ 5 & 46(27) & 29(17) & 15(9) \\ 10 & 1(1) & 2(1) & 0 \\ \text { Not reported } & 3(2) & 0 & 23(14)\end{array}$

(b) Significance level of $\mathrm{Cl}(\%)$

\begin{tabular}{lccl}
90 & $1(1)$ & $14(8)$ & $1(1)$ \\
95 & $14(8)$ & $125(74)$ & 0 \\
97.5 & $4(2)$ & $7(4)$ & 0 \\
Other & 0 & $1(1)$ & 0 \\
Not reported & 0 & 0 & $1(1)$ \\
\hline
\end{tabular}

be justified, but we find that this recommendation is neglected by the majority of authors. It is remarkable that several authors performed only one analysis for the primary outcome and the lack of consistency between the significance level chosen in sample size calculations and the CI reported further highlights confusion of noninferiority trials. Not knowing how to deal with missing data nor appropriate sensitivity analyses, also adds to the confusion. The combination of these recent findings assessed from high-impact journals and the inconsistency in guidelines indicate: (1) the non-inferiority design is not well understood by those using the design and (2) methods for non-inferiority designs are yet to be optimised.

We anticipated that poor reporting of articles would bias towards concluding non-inferiority; however, the poorly reported trials were less likely to demonstrate noninferiority. This is somewhat reassuring. Nevertheless, it is essential to ensure that what is reported at the end of a trial was prespecified before the start of a trial: scientific credibility and regulatory acceptability of a non-inferiority trial rely on the trial being well-designed and conducted according to the design..$^{23}$ It is possible that the quality of a trial may also depend on the quality of the outcome; unresponsive outcomes that miss important differences between treatments may be intentionally or unintentionally chosen to demonstrate non-inferiority. Therefore, it is also important that the outcome chosen is robust.

Almost $80 \%$ of studies concluded non-inferiority, although it is unclear whether this is due to the reporting in articles or publication bias. It appears that positive results (ie, alternative hypotheses) are published more often, regardless of trial design, as this number is consistent with other studies that found that more than $70 \%$ of published superiority trials demonstrated superiority. ${ }^{24} 25$

More than half of articles reported $p$ values, of which approximately a third reported $\mathrm{p}$ values for a two-sided test for superiority. $p$ Values, if reported, should be
Table 6 Reporting of (a) missing data and (b) sensitivity analyses

n (\%)

(a) Imputation performed
Yes
Worst-case scenario
Multiple imputation
Last observation carried forward
Complete case analysis
Best-case scenario
Last observation carried forward and
worst-case scenario
Best-case/worst-case scenario
Mean imputation
Complete case analysis, multiple imputation
using propensity scores and multiple
imputation using regression modelling
Other and worst-case scenario
Other

Not reported

Unclear

Including NEJM protocols $(\mathrm{N}=61)$

Yes

No

Not reported

Unclear

(b) Sensitivity analyses performed Yes

Patient population

Competing risks

Statistical modelling

Adjusted for baseline variables

Excluded protocol violations

On-treatment

Patient population/other

Unclear

Other

Missing data

Best-case/worst-case scenario

Complete case analysis

Imputation of missing values

Multiple imputation

Worst-case scenario

Baseline observation carried forward

Baseline observation carried forward and

complete case analysis

Complete case analysis, multiple imputation

using propensity scores and multiple

imputation using regression modelling

Complete case analysis and missing not at random

Complete case analysis and best-case

scenario

Different methods

Last observation carried forward

3

3

3

3

Modelling

Observed failure

Worst-case scenario and last observation carried forward 


\begin{tabular}{lc} 
Table 6 Continued & \\
\hline & $\mathbf{n}(\%)$ \\
\hline No & $103(61)$ \\
Unclear & $1(1)$ \\
Including NEJM protocols & \\
Yes & $38(62)$ \\
No & $23(38)$ \\
\hline
\end{tabular}

calculated for one-sided tests corresponding to the noninferiority hypothesis; that is, with $\mathrm{H}_{0}$ : $\delta$ =margin. $\mathrm{p}$ Values for superiority should not be presented unless following the demonstration of non-inferiority, where a preplanned superiority hypothesis is tested. ${ }^{26}$

\section{Comparison with other studies}

The value of the non-inferiority margin was almost always reported, but more than half of articles made no attempt to explain how the choice was justified. While justification of the margin is low, this is actually an improvement from Schiller et $a l^{27}$ who reported $23 \%$ articles made a justification, although this difference could be because only high-impact journals were included in this review. There were equally as many articles that planned and reported an ITT analysis compared with articles that performed ITT and PP analyses. This is surprising given that CONSORT 2006 states that an ITT analysis can bias non-inferiority trials towards showing non-inferiority. ${ }^{1}$ These results were lower than found by Wangge et $a l^{28}$ who reported $55 \%$ used either an ITT or PP and $42 \%$ used ITT and PP. Most articles presented two-sided 95\% CIs, which is consistent with results from Le Henanff $e t a l .^{29}$

Table 7 Quality of reporting of trials associated with conclusions of non-inferiority

\begin{tabular}{|c|c|c|c|c|}
\hline \multirow[b]{2}{*}{ Grade } & \multicolumn{4}{|c|}{ Concluded non-inferiority } \\
\hline & $\begin{array}{l}\text { Yes } \\
(\mathrm{N}=132) \\
n(\%)\end{array}$ & $\begin{array}{l}\text { No } \\
(\mathrm{N}=29) \\
\mathrm{n}(\%)\end{array}$ & $\begin{array}{l}\text { Other } \\
(\mathrm{N}=7) \\
n(\%)\end{array}$ & $\begin{array}{l}\text { Total } \\
(\mathrm{N}=168) \\
\mathrm{n}(\%)\end{array}$ \\
\hline Excellent† & $11(73)$ & $2(13)$ & $2(13)$ & 15 \\
\hline Good $\ddagger$ & $55(86)$ & $9(14)$ & $0(0)$ & 64 \\
\hline Fair§ & $48(80)$ & $8(13)$ & $4(7)$ & 60 \\
\hline Poorף & $18(62)$ & $10(34)$ & $1(3)$ & 29 \\
\hline
\end{tabular}

*Excluding trials that concluded 'other': $\chi_{1}^{2}=3.76 ; p=0.05$ (Cochran-Armitage test).

†Excellent if margin justified, $\geq 2$ analyses on patient population performed, type I error rate consistent with significance level of $\mathrm{Cl}$. $\ddagger$ Good if fulfilled two of the following: margin justified, $\geq 2$ analyses on patient population performed, type I error rate consistent with significance level of $\mathrm{Cl}$.

$\S$ Fair if fulfilled one of the following: margin justified, $\geq 2$ analyses on patient population performed, type I error rate consistent with significance level of $\mathrm{Cl}$.

qPoor if margin not justified, $<2$ analyses on patient population performed, type I error rate not consistent with significance level of $\mathrm{Cl}$.
There were very few articles that referred to preserving the treatment effect based on estimates of the standard of care arm from previous trials. It is vital that authors acknowledge this to ensure the standard of care is effective. If the control was to have no effect at all in the study, then finding a small difference between the standard of care and new intervention would be meaningless. ${ }^{2}$

Clinical considerations ${ }^{12911-13}$ to justify the choice of the margin often had inadequate justifications, such as 'deemed appropriate' or 'consensus among a group of clinical experts'. Non-inferiority is only meaningful if it has strong justification in the clinical context and so should be reported. If the justification includes a measurable reduction in adverse events, these should be measured and the benefit should be demonstrated. Guidelines recommend that the choice of margin should be justified primarily on clinical grounds; however, previous trials and historical data should also be considered if available. As an example, Gallagher et $a l^{30}$ justify the choice of the margin providing as much information as possible by including references to all published reports and providing data from the institution where the senior author is based.

A statement often used in articles reviewed was 'the choice of the margin was clinically acceptable'. This statement does not contain enough information to justify the choice of the non-inferiority margin. If the choice of the margin is based on a group of clinical experts, authors should provide information on how many experts were involved and how many considered the choice of the margin being acceptable: a consensus among a group of 3 clinicians from 1 institution is different from a consensus of 20 clinicians representing several institutions. Radford $e t a \hat{l}^{11}$ justify the choice of the non-inferiority margin after performing a delegate survey at a symposium. This method may be a way forward for researchers to obtain clinical assessment from a large group of clinicians. Even better would be to obtain formal assessments, using, for example, the Delphi method, ${ }^{32}$ which has been used in the COMET initiative, ${ }^{33}$ after presenting the proposed research at a conference or symposium for clinicians to really engage with the question at hand.

Definitions provided by authors were inconsistent under what they classed as ITT, PP, mITT and as-treated, for example, "all patients randomised who received at least one dose of treatment" was defined at least once in each classification. According to the guidelines, the PP definition excludes patients from the analysis, but it is unclear what those exclusions are. The ambiguity of how PP is defined was evident in this review as definitions provided by authors could not be succinctly categorised.

Many articles presented only one analysis, despite most guidelines recommending at least two analyses. ${ }^{1} 291012$ Unfortunately, guidelines differ in their advice on which of the two analyses should be chosen to 
base conclusions on. This regrettable, state of affairs was clearly reflected in our review.

The ITT and PP analyses have their biases and so neither can be taken as a 'gold standard' for noninferiority trials. The analysis of the primary outcome is the most important result for any clinical trial. It should be predefined in the protocol what patients should adhere to and should be considered at the design stage what can be carried out to maximise adherence. It should be made clear exactly who is included in analyses given the variety of definitions provided by various authors, particularly for PP analyses where definitions are subjective. Most authors included treatment-related exclusions such as 'received treatment', 'completed treatment' or 'received the correct treatment'. Such differences in definitions may be superficially small, but could in fact make critical differences to the results of a trial.

Poor reporting of whether the hypothesis test was onesided or two-sided or absence of the type I error rate in the sample size calculation meant over a quarter of articles were not clearly consistent with regard to the type I error rate and corresponding CI.

Most guidelines advise presenting two-sided 95\% CIs and this is what most articles presented. However, this recommendation may cause some confusion between equivalence and non-inferiority trials. A 5\% significance level is maintained using 95\% CIs in equivalence trials for twosided hypotheses, whereas non-inferiority takes a onesided hypothesis and so a two-sided $90 \%$ CI should be calculated. If a one-sided type I error rate of $2.5 \%$ is used in the sample size calculation, then this corresponds to the stricter two-sided $95 \%$ CIs, not a one-sided $95 \%$ CI. ${ }^{34}$

The power and type I error rate should be clearly reported within sample size calculations and whether the type I error rate is for a one-sided or two-sided test. For example, the CAP-START trial used a one-sided significance test of 0.05 with two-sided $90 \%$ CIs, and the authors provide exact details of the sample size calculation in online supplementary appendix. ${ }^{35}$ If presenting one bound of the CI throughout an article, this must be performed clearly and consistently as described by Schulz-Schüpke et al, ${ }^{36}$ Lucas et $a l,{ }^{37}$ Gülmezoglu et al. ${ }^{38}$ Recently, JAMA have introduced a policy to present the lower bound of the CI with the upper bound tending towards infinity, ${ }^{39}$ and this has been put into practice in recent non-inferiority trials. ${ }^{40-43}$

It is unclear whether the potential issues surrounding missing data are well recognised for non-inferiority studies, given that the majority of articles did not explicitly state whether or not methods to handle missing outcome data would be considered. Most trials that used multiple imputation stated the number of imputations used but few discussed the assumptions made, which are particularly critical in this context. Some missing data are inevitable, but naive assumptions and/or analysis threaten trial validity for ITT and PP analyses, ${ }^{14}$ particularly in the non-inferiority context where more missing data can bias towards demonstrating non-inferiority. ${ }^{44}$
It is recommended for trials to clearly report whether imputation methods to handle missing data were or were not performed. If imputation was used, it should be clearly stated what method was used along with any assumptions made, following the guidelines of Sterne $e t a^{45}$

Only about a third of articles reviewed reported using sensitivity analyses. There was some confusion between sensitivity analyses for missing data and secondary analyses. Sensitivity analyses for missing data should keep the primary analysis model, but vary the assumptions about the distribution of the missing data, to establish the robustness of inference for the primary analysis to the inevitably untestable assumptions about the missing data. In contrast, secondary analysis with regard to excluding patients for the primary outcome is attempting to answer a separate, secondary question. ${ }^{46}$ Thus, while EMEA 2000 and CONSORT 2012 describe this as sensitivity analysis (and many papers we reviewed followed this), in general this will not be the case, and conflating the two inevitably leads to further confusion.

The focus of the analysis for non-inferiority trials should be on patients who behaved as they were supposed to within a trial, that is the PP population, but rather than excluding patients from the PP analyses, an alternative approach would be to make an assumption about the missing data for patients who do not adhere to the predefined PP definition and then impute missing outcomes for these patients as if they had continued in the trial without deviating. Sensitivity analyses should then be used to check robustness of these results. However, currently, it is unclear what methods are appropriate to achieve this goal.

\section{Subgroup of trials with published protocols}

The mandatory publication of protocols taken from NEJM publications improved results for all criteria assessed. This reiterates the findings from Vale $e t a t^{47}$ who evaluated the risk of bias assessments in systematic reviews assessed from published reports, but had also accessed protocols directly from the trial investigators and found that deficiencies in the medical journal reports of trials does not necessarily reflect deficiencies in trial quality. Given this, it is clear that a major improvement in the reporting of non-inferiority trials would result if all journals followed the practice. Since publication of e-supplements is very cheap, there appears to be no reason not to do this.

\section{Strengths and limitations}

This research demonstrates the inconsistency in the recommendations for non-inferiority trials provided by the available guidelines, which was also reflected within this review. We have provided several recommendations using examples for researchers wishing to use the noninferiority design and have outlined the most important recommendations that we hope will be taken up in future guidelines (box 1). We have also highlighted the importance of missing data and using sensitivity analyses 


\section{Box 1 Recommendations}

- Justification of the margin should be a made mandatory in journals.

- Authors should make reference to preserving the treatment effect based on estimates of the standard-of-care arm from previous trials.

- Presentation of the $\mathrm{Cl}$ should be consistent with the type I error rate used in sample size calculations.

- Analyses should be performed to answer the question of interest (ie, the primary outcome) using additional analyses to test the robustness of that definition, rather than to heedlessly satisfy intention-to-treat and per-protocol definitions.

- Methods to handle missing data should be considered, and sensitivity analyses should be considered to test the assumptions of missing data made on the primary analysis.

- Protocols should always be published as online supplementary material and authors should make use of online supplementary material to include additional detail on methods (such as details for justifying the choice of the non-inferiority margin and full definition of analyses conducted), so that a word limit for a published article should not be an excuse for poor reporting.

specific to non-inferiority trials. There are also some limitations in this review. First, a justification of the choice of the margin was recorded as such if any attempt was made to do so. Therefore, one could argue that inadequate attempts were counted as a 'justification'; however, there was good agreement between reviewers when independently assessed. Second, only one reviewer extracted information from all articles and therefore assessments may be subjective. However, there was good agreement when a random $5 \%$ of papers were independently assessed, and the categorisation of the justification of the non-inferiority margin was also independently assessed in all papers where a justification was given. Third, an update of the CONSORT statement for non-inferiority trials was published during the period of the search in $2012,{ }^{9}$ which could improve the reporting of non-inferiority trials over the next few years. However, the first CONSORT statement for noninferiority trials published in $2006^{1}$ was released well before the studies included in our search and we have found that reporting of non-inferiority trials remains poor.

\section{CONCLUSION}

Our findings suggest clear violations of available guidelines, including the CONSORT 2006 statement (published 4 years before the first paper in our review), which concentrate on improving how non-inferiority trials are reported and is widely endorsed across medical journals.

There is some indication that the quality of reporting for non-inferiority studies can affect the conclusions made and therefore the results of trials that fail to clearly report the items discussed above should be interpreted cautiously. It is essential that justification for the choice of the non-inferiority margin becomes standard practice, providing the information early on when planning a study including as much detail as possible. If the choice of the non-inferiority margin changes following approval from an ethics committee, justification for the change and changes to the original sample size calculation should be explicit. If journals enforced a policy where authors must justify the choice of the noninferiority margin prior to accepting publication, this would encourage authors to provide robust justifications for something so critical given that clinical practice may be expected to change if the margin of noninferiority is met.

Sample size calculations include consideration of the type I error rate, which should be consistent with the CIs as these provide inferences made for non-inferiority when compared against the margin. Inconsistency between the two may distort inferences made, and stricter CIs may lack power to detect true differences for the original sample size calculation. If any imputation was performed, then this should be detailed along with its underlying assumptions, supplemented with sensitivity analyses under different assumptions about the missing data. There is an urgent need for research into appropriate ways of handling missing data in the PP analysis for non-inferiority trials; once resolved, this analysis should be the primary analysis.

Information that is partially prespecified before the conduct of a trial may inadvertently provide opportunities to modify decisions that were not prespecified at the time of reporting without providing any justification. It is therefore crucial for editors to be satisfied that criteria are defined a priori. A compulsory requirement from journals to publish protocols as e-supplements and even statistical analysis plans along with the main article would avoid this ambiguity.

\section{Author affiliations}

${ }^{1}$ MRC Clinical Trials Unit at UCL, Institute of Clinical Trials and Methodology, London, UK

${ }^{2}$ MRC Clinical Trials Unit at UCL, London Hub for Trials Methodology Research, London, UK

${ }^{3}$ MRC Tropical Epidemiology Group, Department of Infectious Disease Epidemiology, London School of Hygiene \& Tropical Medicine, London, UK ${ }^{4}$ Department of Medical Statistics, London School of Hygiene \& Tropical Medicine, London, UK

Acknowledgements SR is supported by a Medical Research Council PhD studentship (grant number 1546500). TPM and JRC are supported by the Medical Research Council CTU at UCL (grant numbers 510636 and 532196).

Contributors SR conceived the study, carried out data extraction and analysis, and wrote the manuscript. TPM performed data extraction and critical revision of the manuscript. KF and JRC helped in critical revision of the manuscript. PPJP conducted data extraction, analysis and critical revision of the manuscript.

Funding This research received no specific grant from any funding agency in the public, commercial or not-for-profit sectors.

Competing interests None declared. 
Provenance and peer review Not commissioned; externally peer reviewed.

Data sharing statement No additional data are available.

Open Access This is an Open Access article distributed in accordance with the terms of the Creative Commons Attribution (CC BY 4.0) license, which permits others to distribute, remix, adapt and build upon this work, for commercial use, provided the original work is properly cited. See: http:// creativecommons.org/licenses/by/4.0/

\section{REFERENCES}

1. Piaggio G, Elbourne DR, Altman DG, et al. Reporting of noninferiority and equivalence randomized trials: an extension of the CONSORT statement. JAMA 2006;295:1152-60.

2. Food and Drug Administration, HHS. Draft Guidance for Industry Non-Inferiority Clinical Trials. 2010.

3. Schumi J, Wittes JT. Through the looking glass: understanding non-inferiority. Trials 2011;12:106

4. Treadwell JR, Uhl S, Tipton $\mathrm{K}$, et al. Assessing equivalence and noninferiority. J Clin Epidemiol 2012;65:1144-9.

5. Snapinn SM. Noninferiority trials. Curr Control Trials Cardiovasc Med 2000;1:19-21.

6. Murthy VL, Desai NR, Vora A, et al. Increasing proportion of clinical trials using noninferiority end points. Clin Cardiol 2012;35:522-3.

7. Suda KJ, Hurley AM, McKibbin T, et al. Publication of noninferiority clinical trials: changes over a 20 -year interval. Pharmacotherapy 2011;31:833-9.

8. Vermeulen L. Gain in popularity of noninferiority trial design: caveat lector. Pharmacotherapy 2011;31:2.

9. Piaggio G, Elbourne DR, Pocock SJ, et al. Reporting of noninferiority and equivalence randomized trials: extension of the CONSORT 2010 statement. JAMA 2012:308:2594-604.

10. Committee for Medicinal Products for Human Use (CHMP) CPMP Points to consider on switching between superiority and non-inferiority. London, UK, 2000 (cited 3 November 2015). http:// www.ema.europa.eu/docs/en_GB/document_library/Scientific_ guideline/2009/09/WC500003658.pdf

11. Committee for Medicinal Products for Human Use, Efficacy Working Party, Committee for Release for Consultation. Committee for Medicinal Products for Human Use (CHMP) guideline on the choice of the non-inferiority margin. Stat Med 2006;25:1628-38.

12. International Conference on Harmonisation; guidance on statistical principles for clinical trials; availability-FDA. Notice. Fed Regist 1998;63:49583-98.

13. Food, Drug Administration HHS. International Conference on Harmonisation; choice of control group and related issues in clinical trials; availability. Notice. Fed Regist 2001;66:24390-1.

14. Chan AW, Tetzlaff JM, Gøtzsche PC, et al. SPIRIT 2013 explanation and elaboration: guidance for protocols of clinical trials. BMJ 2013;346:e7586.

15. GAO. New drug approval. FDA's Consideration of Evidence from Certain Clinical Trials. 2010 (cited 1 April 2016). http://www.gao.gov/ assets/310/308301.pdf

16. Moher D, Hopewell S, Schulz KF, et al. CONSORT 2010 explanation and elaboration: updated guidelines for reporting paralle group randomised trials. BMJ 2010;340:c869.

17. D'Agostino RB Sr, Massaro JM, Sullivan LM. Non-inferiority trials: design concepts and issues-the encounters of academic consultants in statistics. Stat Med 2003;22:169-86.

18. Matsuyama Y. A comparison of the results of intent-to-treat, per-protocol, and g-estimation in the presence of non-random treatment changes in a time-to-event non-inferiority trial. Stat Med 2010;29:2107-16.

19. ICH Harmonised Tripartite Guideline. Statistical principles for clinical trials. International Conference on Harmonisation E9 Expert Working Group. Stat Med 1999;18:1905-42.

20. Abraha I, Montedori A. Modified intention to treat reporting in randomised controlled trials: systematic review. BMJ 2010;340: c2697.

21. ISI Web of Knowledge (cited 31 May 2015). http://admin-apps. webofknowledge.com/JCR/JCR

22. Rubin DB. Multiple imputation for nonresponse in surveys. New York: John Wiley and Sons, 1987.
23. Hwang IK, Morikawa T. Design issues in noninferiority equivalence trials. Drug Inform J 1999;33:1205-18.

24. Vickers A, Goyal N, Harland R, et al. Do certain countries produce only positive results? A systematic review of controlled trials. Control Clin Trials 1998;19:159-66.

25. Hopewell S, Loudon K, Clarke MJ, et al. Publication bias in clinical trials due to statistical significance or direction of trial results. Cochrane Database Syst Rev 2009;(1):MR000006.

26. Tunes da Silva G, Logan BR, Klein JP. Methods for equivalence and noninferiority testing. Biol Blood Marrow Transplant 2009;15(1 Suppl):120-7.

27. Schiller P, Burchardi N, Niestroj M, et al. Quality of reporting of clinical non-inferiority and equivalence randomised trials-update and extension. Trials 2012;13:214.

28. Wangge $\mathrm{G}$, Klungel $\mathrm{OH}$, Roes $\mathrm{KC}$, et al. Room for improvement in conducting and reporting non-inferiority randomized controlled trials on drugs: a systematic review. PLoS ONE 2010;5:e13550.

29. Le Henanff A, Giraudeau B, Baron G, et al. Quality of reporting of noninferiority and equivalence randomized trials. JAMA 2006;295:1147-51.

30. Gallagher TQ, Hill C, Ojha S, et al. Perioperative dexamethasone administration and risk of bleeding following tonsillectomy in children: a randomized controlled trial. JAMA 2012;308:1221-6.

31. Radford J, Illidge T, Counsell N, et al. Results of a trial of PET-directed therapy for early-stage Hodgkin's lymphoma. N Engl J Med 2015;372:1598-607.

32. Hasson F, Keeney S, McKenna H. Research guidelines for the Delphi survey technique. J Adv Nurs 2000;32:1008-15.

33. COMET Initiative [cited 2016 July 20th]. http://www.comet-initiative. org/

34. Lesaffre E. Superiority, equivalence, and non-inferiority trials. Bull NYU Hosp Jt Dis 2008:66:150-4.

35. Postma DF, van Werkhoven $\mathrm{CH}$, van Elden LJ, et al. Antibiotic treatment strategies for community-acquired pneumonia in adults. N Engl J Med 2015;372:1312-23.

36. Schulz-Schüpke S, Helde S, Gewalt S, et al. Comparison of vascular closure devices vs manual compression after femoral artery puncture: the ISAR-CLOSURE randomized clinical trial. JAMA 2014;312:1981-7.

37. Lucas BP, Trick WE, Evans AT, et al. Effects of 2- vs 4-week attending physician inpatient rotations on unplanned patient revisits, evaluations by trainees, and attending physician burnout: a randomized trial. JAMA 2012;308:2199-207.

38. Gülmezoglu AM, Lumbiganon $\mathrm{P}$, Landoulsi $\mathrm{S}$, et al. Active management of the third stage of labour with and without controlled cord traction: a randomised, controlled, non-inferiority trial. Lancet 2012;379:1721-7.

39. Kaji $\mathrm{AH}$, Lewis RJ. Noninferiority trials: is a new treatment almost as effective as another? JAMA 2015;313:2371-2

40. Lee $\mathrm{CH}$, Steiner $\mathrm{T}$, Petrof $\mathrm{EO}$, et al. Frozen vs fresh fecal microbiota transplantation and clinical resolution of diarrhea in patients with recurrent Clostridium difficile infection: a randomized clinical trial JAMA 2016;315:142-9.

41. Rahman NM, Pepperell J, Rehal S, et al. Effect of opioids vs NSAIDs and larger vs smaller chest tube size on pain control and pleurodesis efficacy among patients with malignant pleural effusion: the TIME1 randomized clinical trial. JAMA 2015;314:2641-53.

42. Stevenson AR, Solomon MJ, Lumley JW, et al. Effect of laparoscopic-assisted resection vs open resection on pathological outcomes in rectal cancer: the ALACART randomized clinical trial. JAMA 2015;314:1356-63.

43. Fleshman J, Branda M, Sargent DJ, et al. Effect of laparoscopic-assisted resection vs open resection of stage II or III rectal cancer on pathologic outcomes: the ACOSOG Z6051 randomized clinical trial. JAMA 2015;314:1346-55.

44. Wiens BL, Rosenkranz GK. Missing data in noninferiority trials. Stat Biopharm Res 2013;5:383-93.

45. Sterne JA, White IR, Carlin JB, et al. Multiple imputation for missing data in epidemiological and clinical research: potential and pitfalls. BMJ 2009;338:b2393.

46. Morris TP, Kahan BC, White IR. Choosing sensitivity analyses for randomised trials: principles. BMC Med Res Methodol 2014;14:11.

47. Vale CL, Tierney JF, Burdett S. Can trial quality be reliably assessed from published reports of cancer trials: evaluation of risk of bias assessments in systematic reviews. BMJ 2013;346:f1798. 